\title{
Quality of Life among Egyptian Migrants Living in Australia
}

\author{
Endrawes $\mathrm{G}^{1 *}$ and Fernandez $\mathrm{R}^{2}$ \\ ${ }^{1}$ School of Nursing and Midwifery, University of Western Sydney, Australia \\ ${ }^{2}$ School of Nursing, Faculty of Health and Behavioural Sciences, University of \\ Wollongong, Australia
}

*Corresponding author: Gihane Endrawes, School of Nursing and Midwifery, College

\section{Research Article}

Volume 3 Issue 5

Received Date: October 14, 2019

Published Date: October 29, 2019

DOI: $10.23880 /$ nhij-16000206

of Health \& Science, University of Western Sydney, Locked Bag 1797, Penrith South DC NSW 1797, Australia, Tel: 0400027345; Email: g.endrawes@westernsydney.edu.au

\section{Abstract}

The aim of this study was to identify the predictors of QoL of migrant Egyptians living in Australia. Participants' perception of their QoL was assessed using the modified Flanagan Quality of Life Scale (QoLS). Cross-cultural adaptation was undertaken of the English version of the QoLS. The QoLS was self-administered to 200 Egyptian migrants. Cronbach's alpha coefficient (to test reliability) was 0.96 for the total of QoLS and $0.84,0.78$ and 0.66 for the 'Relationships and Material Wellbeing' 'Personal, Social and Community Commitment' and 'Health and Functioning' subscales respectively. The mean QoL score was 81.6 ( $\mathrm{SD} \pm 15.6$ ). Of the five socio-demographic factors investigated employment status was the factor most strongly associated with high QoL $(\mathrm{P}=0.005)$. This study is the first to demonstrate the impact of employment status on the QoL of Egyptian migrants in Australia. Culturally sensitive interventions directed towards addressing this issue need to be implemented.

Keywords: Nursing; Population; Migrants; Demographic

\section{Introduction}

\section{Background}

Over the past 25 years, globally, there has been acceleration in human migration rates to developed countries with the number of international migrants estimated at 214 million in 2010 [1]. Contemporary migration is stimulated by various factors including economic, social, political or environmental factors, [2] however the underlying intention irrespective of the reason for migration is to have a better life style and improved quality of life [3].
QoL is a multidimensional concept which is hard to define or measure as it is determined by ethical, cultural, personal, physical and religious factors [4]. QoL has been largely associated with physical and mental health and social functioning particularly in migrant populations [58].

It has been well established that migrants particularly those who are from culturally and linguistically diverse backgrounds (CALD) are at higher risk of having poorer quality of life than the general population due to language barriers and sociocultural differences $[9,10]$. In a study undertaken on 176 elderly Iranians in Sweden, the QoL of 


\section{Nursing \& Healthcare International Journal}

Iranians in Sweden was similar to that of their countrymen in Iran but differed from that of Swedes who reported a higher QoL than Iranians [11].

\section{Factors Affecting Qol in Migrants}

Various factors including migrants' demographic profile and employment status affect their QoL [12]. Results from a study undertaken on 253 Iraqi migrants in Malaysia indicated a significantly higher Qol relating to physical function and general health among males [13]. In addition higher physical function and lower mental health was reported among younger migrants [13]. Furthermore, Poppe, Crombez, Hanoulle, Vogelaers and Petrovic [14] explored the relationship between personality traits and coping and its impact on QoL. They found that personality and coping are related and influenced both physical and mental health QoL. This finding is supported by other research [15-17].

Marital status is also associated with QoL with married migrants reporting higher QoL compared to their unmarried counterparts $[13,18]$. The level of education has also been reported to be a predictor of QoL with lower education levels associated with poorer QoL among migrants $[13,19]$. Similarly unemployment was found to negatively affect QoL with people reporting lower subjective well-being when unemployed Khan, et al. [20] the length of residency in the adopted country has also been identified as affecting the QoL of migrants, however the evidence remains controversial [11,21]. Some studies have reported a positive association between longer period of residence and social functioning and role limitation aspects of quality of life among women [11] while others have indicated that long term residency were predictors of lower physical health [21].

Higher education, economic status and living with others were positively correlated with higher QoL [22]. Economic status was the most significant predictor for better or poorer physical and mental QoL. One study [23] explored the effects of education on the QoL among elderly Spanish community population found a strong association between education and higher QoL, while lower educational level was associated with unhappiness and poor social relationships which directly affects QoL. Similar findings have been reported by researchers in the general/healthy Norwegian population [24].

There is also controversy in the literature about the QoL of migrants with some experts reporting better QoL among migrants who have relocated to a country that has a higher human development index [11] which includes longer life expectancy, decent standard of living and higher education levels [25] as well as access to health care services [11]. For example, the QoL among a group of Turkish immigrants in Sweden (Very high human development index) was found to be better when compared with Turkish people living in Turkey [9]. Australia has been rated as having a very high human development index second only to Norway [25] therefore it could be expected that migrants living here have a higher QoL. However, there is limited literature on the QoL of migrants, in particular among Egyptian communities living in Australia.

\section{Egyptians in Australia}

According to the Australian 2011 Census, 36,532 Australian residents declared that they were born in Egypt of which the majority lived in Sydney NSW. Egyptian migrants are highly educated with more than a third (36.8\%) having a degree or a higher qualification which is more than double that of the general Australian population [26].

Extensive research has been undertaken on QoL of people with chronic diseases [27] however, few studies have assessed the QoL among the general migrant population, especially among people from CALD backgrounds [10]. Therefore, the aim of this study was to investigate the QoL and the socio demographic predictors of QoL among adult Egyptian migrants living in Australia.

\section{Method}

\section{Study Setting}

This cross-sectional study was conducted across three local Egyptian Coptic churches in a large metropolitan city in Australia. Churches have often been used in previous research studies and have been reported to have powerful influence on health related behaviour change such as smoking cessation, breast cancer screening, diabetes prevention, nutrition and physical activity [28-32] for diverse communities.

\section{Sample}

A convenience sample of migrant Egyptian men and women aged 18 years and over and living in Australia were eligible to participate in the study. Participants in this cross sectional study were people who attended Egyptian Coptic churches and were able to read either English or Arabic. 


\section{Nursing \& Healthcare International Journal}

\section{Data Collection}

Data were collected relating to participants' demographics and their perception of their QoL. The demographic data collected included: gender, age, employment status, marital status, type of employment and religious/spiritual orientation. Participants' perception of their QoL was assessed using the modified Flanagan Quality of Life Scale (QoLS) [33]. This scale was chosen as it encompasses a wide range of aspects including relationships, material comfort, health, recreation and work satisfaction which have a major impact on the QoL of migrants [24,34]. The modified QoLS is a 16-item self-administered instrument which takes approximately five minutes to complete. The 16 items are categorized under three subscales which assesses relationships and material wellbeing, health and functioning and personal, social and community commitment. The instrument is rated on a 7-point scale as: delighted, pleased, mostly satisfied, mixed, mostly dissatisfied, unhappy and terrible, responses ranging from 7 for delighted and 1 for terrible [35]. The QoLS scores range from 16 to 112 , maximum score obtainable is 112. A higher score indicates higher QoL.

The scale has been used among healthy populations as well as with populations with physical or mental health conditions. The QoL scores for healthy population have been reported to be 90 , and for other conditions it ranged from 61 for Israeli patients with post-traumatic stress disorder, to 70 for fibromyalgia, to 82 for psoriasis, urinary incontinence and chronic obstructive pulmonary disease [33]. The QoL scale has been shown to have high reliability and validity in CALD groups [24,33,34]. This scale was originally developed in English and is translated in Swedish, Norwegian, Hebrew, Spanish, Farsi, Portuguese, Italian, German, French, Greek, Islandic, Danish, Turkish, Chinese and Thai [33].

\section{Translation of the QoL Scale}

The English version of the QoLS is available in the public domain, however, permission to use the scale was sought from the developers. At the time of conducting the study, the QoLS was not available in the Arabic language therefore the scale was translated into Arabic (QoLS-A). Translation of the QoLS was conducted according to recommended guidelines Guillemin, et al. [36] by a professional accredited translator and back translated to English to ensure that the meaning was not lost in the process of translation. The back-translation was performed by a bilingual expert who had not seen the scale before.

\section{Procedure}

Approval from the University Ethics Committee was obtained prior to the conduct of the study. The researchers informed the church leaders about the study and sought their permission and support to conduct the study in the churches. An announcement was made by the church leader at the end of the church service informing people about the study and inviting them to participate. The first author was available outside the church to provide further information about the study for those who were willing to participate. Those who agreed to participate received a participant information sheet and questionnaire. Participants were requested to complete the questionnaire and place it in a secure box located in the church. The secure box could be accessible at any time of the day or night from outside the church. Both English and Arabic versions of the QoLS were used, depending on participants' preference of either language. Returning the completed surveys implied that participants have agreed voluntarily to participate in the study.

\section{Statistical Analysis}

All data were entered and analysed using SPSS 17 for windows. Accuracy of the data entry was checked prior to analysis by computing frequencies to detect incorrect entries [37]. Internal consistency determined by Cronbach's alpha was used to assess the reliability of QoLS. The alpha was accepted as adequate if it was greater than 0.70 . Pearson correlation has been undertaken to test the relationship between age, gender, marital status, language in which the survey was completed and employment status and QoL. Significance level was accepted at $p<0.05$. Results are presented using frequencies and means where appropriate.

\section{Results}

A total of 200 questionnaires were distributed and 156 completed surveys (male: 89; females: 67) were returned for a response rate of $78 \%$. Fifty-nine participants completed the survey in English and 97 in Arabic. The mean age of the participants was 50.64 (SD \pm 9.6 ) (age range 28-73) and the majority (94\%) were married. Employment status was used as a predictor of socioeconomic status. Three quarters $(n=119)$ of the participants were employed and more than half of them had jobs as either health or non-health professionals $(n=$ 83) (Table 1). 


\begin{tabular}{|c|c|}
\hline Age Mean (SD & $\begin{array}{c}\text { 52.10 (SD=9.9) Age Range } \\
\text { (28-73) Frequency (\%) }\end{array}$ \\
\hline \multicolumn{2}{|c|}{ Gender } \\
\hline Male & $89(57)$ \\
\hline Female & $67(43)$ \\
\hline \multicolumn{2}{|c|}{ Employment status } \\
\hline Employed & $119(76)$ \\
\hline Unemployed & $2(3)$ \\
\hline Retired & $33(21)$ \\
\hline Questionnaire completed in \\
\hline English & $59(38)$ \\
\hline Arabic Marital status \\
\hline \multicolumn{2}{|c|}{$97(62)$} \\
\hline Living with a partner & $146(94)$ \\
\hline Not living with a partner & $10(6)$ \\
\hline
\end{tabular}

Table 1: Demographic characteristics of participants $(\mathrm{n}=156)$.

\section{Reliability of the QoL Scale}

The internal consistency of the scale was high (Cronbach's alpha coefficients of 0.96) for the total of QoLS. The Cronbach's alpha for the 'Relationships and Material Wellbeing' subscale was 0.84, 'Personal, Social and Community Commitment' subscale was 0.78 and the 'Health and Functioning' subscale was 0.66.

\section{Quality of Life}

The mean total QoL score for all participants was 81.6 $( \pm 15.6)$. Mean scores for the 'Relationship and Material Wellbeing' subscales was 36.8 ( \pm 7.5); Personal, Social and Community Commitment' subscale was 24.8 ( \pm 5.7 ) and 'Health and Functioning' subscale was 20 ( \pm 4.2). Mean scores and SD for each item on the scale are reported in Table 2. The total QoL scores among those who completed the questionnaire in English were higher $(84.0 \pm 13.8)$ than those who completed the questionnaire in Arabic (80.1 \pm 16.5$)$ but these total QoL scores results were not statistically significant ( $\mathrm{p}=0.141$ ). However, $\mathrm{QoL}$ scores for two items including Socializing: meeting other people, doing things and Reading, music, or watching entertainment from the 'Relationships and Material Wellbeing' subscale were significantly higher $(\mathrm{p}<0.05)$ among those who completed the questionnaire in English compared to those who completed the questionnaire in Arabic. Similar results were obtained for the item participating in active recreation from the 'Personal Social and Community Commitment' subscale.

\begin{tabular}{|c|c|c|c|c|}
\hline Subscale & QoLS Items & $\begin{array}{l}\text { Mean Score } \\
\text { (SD) }\end{array}$ & English & Arabic \\
\hline \multirow{7}{*}{$\begin{array}{l}\text { Relationships and material } \\
\text { wellbeing }\end{array}$} & $\begin{array}{l}\text { Material comforts: home, food, modern } \\
\text { conveniences, and financial security }\end{array}$ & $5.3(1.3)$ & $5.48(1.0)$ & $5.1(1.4)$ \\
\hline & $\begin{array}{l}\text { Relationships with parents, sibling and other } \\
\text { relatives: communicating, visiting, helping }\end{array}$ & $5.4(1.4)$ & $5.42(1.4)$ & $5.3(1.4)$ \\
\hline & Having and rearing children & $5.4(1.7)$ & $5.4(1.5)$ & $5.3(1.7)$ \\
\hline & $\begin{array}{l}\text { Close relationship with spouse or other } \\
\text { significant other }\end{array}$ & $5.5(1.6)$ & $5.5(1.3)$ & $5.4(1.6)$ \\
\hline & Close friends & $5.1(1.5)$ & $5.2(1.3)$ & $5.1(1.5)$ \\
\hline & Socializing: meeting other people, doing things & $5.0(1.4)$ & $* 5.3(1.2)$ & $4.8(1.5)$ \\
\hline & Reading, music, or watching entertainment & $5.1(1.4)$ & $* 5.4(1.1)$ & $4.8(1.5)$ \\
\hline Subtotal score & & $36.8(7.5)$ & & \\
\hline \multirow{5}{*}{$\begin{array}{l}\text { Personal Social and } \\
\text { Community commitment }\end{array}$} & $\begin{array}{c}\text { Helping and encouraging others, participating in } \\
\text { organizations, volunteering }\end{array}$ & $5.1(1.5)$ & $5.0(1.4)$ & $5.1(1.4)$ \\
\hline & $\begin{array}{c}\text { Participating in political organizations or public } \\
\text { affairs }\end{array}$ & $4.2(1.9)$ & $4.4(1.7)$ & $4.0(1.9)$ \\
\hline & $\begin{array}{c}\text { Learning: attending school, improving } \\
\text { knowledge }\end{array}$ & $4.9(1.6)$ & $5.2(1.2)$ & $4.7(1.6)$ \\
\hline & $\begin{array}{c}\text { Understanding yourself: knowing what life is } \\
\text { about }\end{array}$ & $5.4(1.4)$ & $5.6(1.0)$ & $5.3(1.4)$ \\
\hline & Expressing yourself creatively & $5.1(1.4)$ & $5.2(1.2)$ & $4.9(1.4)$ \\
\hline \multirow[t]{3}{*}{ Subtotal score } & & $24.8(5.7)$ & & \\
\hline & Health: being physically fit and vigorous & $4.9(1.4)$ & $5.0(1.1)$ & $4.7(1.4)$ \\
\hline & Work: job or home & $5.2(1.5)$ & $5.1(1.5)$ & $5.2(1.4)$ \\
\hline
\end{tabular}

Endrawes G and Fernandez R. Quality of Life among Egyptian Migrants Living in Australia. Nurs Health Care Int J 2019, 3(5): 000206. 


\section{Nursing \& Healthcare International Journal}

\begin{tabular}{|c|c|c|c|c|}
\hline \multirow{2}{*}{ Health and Functioning } & Participating in active recreation & $4.5(1.6)$ & $* 4.8(1.2)$ & $4.3(1.8)$ \\
\cline { 2 - 5 } & Independence, being able to do things yourself & $5.4(1.5)$ & $5.5(1.3)$ & $5.3(1.5)$ \\
\hline Subtotal score & & $20.0(4.2)$ & & \\
\hline Total QoL score & & $81.6(15.6)$. & $84.0(13.8)$ & $80.1(16.5)$ \\
\hline
\end{tabular}

$\mathrm{p}>0.05$ Group Statistics

Table 2: QoL Scores.

\section{Socio-Demographic Predictors of QoL}

Of the five socio-demographic factors investigated, employment status was the factor most strongly associated with high QoL ( $\mathrm{p}=0.005)$. No significant associations were found between QoL and gender, marital status, age and language in which the survey was completed.

\section{Discussion}

There is a extant literature on the QoL of migrants $[11,13,38]$ however there are no studies that assess the QoL of migrant Egyptians. To the best of our knowledge this is the first study investigating the QoL of migrant Egyptians living in Australia. The major strength of this study was the rigor in which it was conducted. Firstly, the translation of the modified Flanagan QoLS was undertaken using published guidelines. Secondly, to minimise bias participants were asked to complete the questionnaire in their own time and place it in the survey return box that was available 24 hours of the day. Finally, all data were entered by an assistant who was not involved with the project.

The mean QoL scores in this study was 81.6 (15.6) which is lower than other studies that have reported QoL scores ranging from 83.1 (12.5) [28], to 90 [33] using the same scale in healthy populations. This result is not surprising as our study was undertaken in a migrant population in comparison to the other studies that were undertaken in a non-migrant population [34]. In addition, the differences in the self-reported QoL in this study may be due to the varying perceptions of health which are influenced by one's culture, language and ethical, personal and religious values.

What is interesting is that scores for Socialising meeting other people, doing things were significantly higher among participants who completed the questionnaire in English compared to those who completed the questionnaire in Arabic. This result is consistent with published literature on acculturation reflects how migrants' social life is negatively affected by migration $[13,39,40]$ where language barrier limits them from socialisation and participating in recreational or leisure activities. Possible explanations for this result could be due to the fact that the majority of participants worked in either professional or semi-professional jobs (such as teachers, engineers, social workers and accountants) which, requires the acquisition of English language skills. Being educated, and having good English language skills, may have facilitated interactions and engagements with others; hence participants were more likely to be active in recreation and socialisation.

Similarly scores for participation in active recreation were significantly higher among participants who completed the questionnaire in English compared to those who completed the questionnaire in Arabic. Culture difference and acculturation level may have played a role, especially for those participants who completed the survey in Arabic. They could have been more likely to have migrated to Australia at an older age and may have still maintaining all or some of their own cultural practices, values and beliefs which may be different from those of the Australian culture. Though the acculturation level of participants was not examined in this study, it could be a possible reason for lower participation in active recreation among migrant Egyptians. Another plausible explanation could be that older migrants rely on family and, social gatherings rather than participating in active recreation [41].

Reading, listening to music and being engaged in activities can be stimulating, enriching, and rewarding thus improving mental well-being and QoL [42]. Scores relating to these aspects were also significantly higher among participants who completed the questionnaire in English compared to those who completed the questionnaire in Arabic. This could be due to the limited reading levels of the participants as well as the lack of culturally related music and entertainment.

These results reflect the importance of socialisation, entertainment, being around people and being engaged in recreational activities, reading and music to the Egyptian person living in Australia. These activities can have a positive effect on mental and physical well-being of 


\section{Nursing \& Healthcare International Journal}

participants, which impacts overall on QoL. Therefore, strategies to encourage migrant Egyptians to participate in active as well as passive recreation such as the development of culturally specific community centres, recreational or leisure clubs are urgently needed as this can have an impact on their quality of life.

In this study, employment status was the only predictor of QoL with retired people reporting poorer QoL. Employment status was a predictor of higher QoL as it is associated with material and well-being, relationships. This result is consistent with previous research where employment status influenced QoL, regardless of the existence of a physical or mental health problem $[14,24,43]$. This result could be due to the fact that for Egyptian migrants, work is important and that retired people feel that they do not contribute to society. In addition, being employed allows individuals to socialise with others, thus, reducing social isolation. Upon retirement, individuals are faced by different changes and losses such as loss of one's income, status and social networks, all of these may contribute to low self-esteem, poorer mental health and overall QoL. It is therefore important to develop culturally sensitive strategies where retired people are able to contribute in some way to society.

Gender, was not identified as a predictor of QoL which is in contrast with other studies where women reported poorer QoL $[13,22]$. This could be due to the fact that females in this study had a stable employment, hence financial security. Financial security has been reported in the literature as having a significant positive impact on the QoL of people [22].

Similarly, age was not identified as a predictor of QoL. This result is also in contrast with other literature that indicates that older people have poorer QoL [44]. It could be postulated that cultural factors such as family support and emotional support in the form of companionship could have contributed to this outcome. Culturally, elderly Egyptians are well respected in their community and are cared for and appreciated by their family and friends hence could have reported better QoL. In addition, as participants in this study were those attending the local Coptic churches it is also possible that religion added meaning to their life resulting in a good QoL for the older Egyptians [45].

Although we did not investigate length of stay and language proficiency in this study, there is extant literature indicating that these two factors play a role in
QoL of migrants $[10,39,46]$. In the current study, language in which the survey was completed was used as a surrogate for English language proficiency among the participants. The results indicated that language was not a predictor of the overall QoL which is in direct contrast to the existing literature on CALD migrants where poor English proficiency was associated with poorer health outcomes and related poorer QoL [46]. Various explanations are possible for this result. Firstly, approbation of their own language and the opportunity to opportunity to complete the survey in their own language could be important to them personally. Secondly, completing the survey in Arabic did not indicate that participants lacked English language proficiency particularly as the majority were employed which require basic language skills to communicate effectively. Finally, participants were highly educated which could have assisted in developing skills that could negate the effect of poor English skills.

Evidence suggests that there is association between employment status and mental health with poorer mental health among those unemployed [47]. It could be postulated that participants in this study who were unemployed may have an underlying mental health issue that is unrecognised. Therefore, there is a need for raising community awareness of mental health issues, early identification and early interventions to reduce the likelihood of developing mental health problems in healthy migrants.

\section{Limitations}

Despite the evidence obtained in this study, the limitations inherent in undertaking such research need to be considered. Firstly, the subjects recruited in this study were from three local Coptic churches hence the results can be generalised to only Coptic Egyptians who make up 87\% of all Egyptians living in Australia [26].

Another limitation of this study is that the responses could be due social desirability bias [48], which may have caused some subjects to over report their quality of life, which is common among certain cultural groups [49].

\section{Implications for Future Research}

In this study participants were not asked their level of education and the type of job was used as a surrogate indicator of qualifications. Future studies need to assess qualifications. Employment status is correlated with better QoL. Further studies are needed to investigate the 


\section{Nursing \& Healthcare International Journal}

type of jobs of retired people to see if type of jobs has an effect on QoL of migrants. Further studies need to be done on other Egyptian groups such as Egyptian Muslims to examine the role of religion and/or spirituality on QoL.

There is a lack of important variables such as data on quality of life, social class and income before immigration, which could be a factor influencing the result of this study, depending on whether participants had poorer or better QoL before immigration. Other data on length of stay in Australia or period of residency and the acculturation level was not collected/unknown, which could also be a factor influencing results. Hence future research is needed to address the impact of these other variables on QoL.

More research is also needed to assess QoL before and after immigration and the impact of migration on QoL among Egyptian groups and other CALD groups. Findings of this study will add to the body of literature and inform health care professionals, policy makers and researchers of potential issues affecting this migrant group. Understanding QoL is critical in order to reduce the health care disparities and provide migrants with a QoL equivalent to the native population.

\section{Conclusion}

This study has demonstrated the impact of employment status on the QoL of Egyptian migrants in Australia. This important factor should be regarded as a priority and culturally sensitive interventions and resources directed towards addressing this issue need to be implemented.

\section{References}

1. IOM (2010) World Migration Report 2010-The Future of Migration: Building Capacities for Change, International Organization for Migration, pp: 3-272.

2. Levitt P, Jaworsky BN (2007) Transnational migration studies: Past developments and future trends. Annu Rev Sociol 33: 129-56.

3. Castles S (2010) Understanding global migration: a social transformation perspective. Journal of Ethnic and Migration Studies 36(10): 1565-1586.

4. Koen M, Foreman MD, Hendrickx A, Godderis J, Abraham IL, et al. (2005) Psychometric properties of the Flemish translation of the NEECHAM Confusion Scale. BMC psychiatry 5(1): 16.
5. Endrawes GL, O Brien, Wilkes L (2007) Mental illness and Egyptian families. International Journal of Mental Health Nursing 16(3): 178-187.

6. Kirmayer LJ, Narasiah L, Munoz M, Rashid M, Ryder AG, et al. (2011) Common mental health problems in immigrants and refugees: general approach in primary care. CMAJ 183(12): E959-E67.

7. Missinne S, Bracke P (2012) Depressive symptoms among immigrants and ethnic minorities: a population based study in 23 European countries. Soc Psychiatry Psychiatr Epidemiol 47(1): 97-109.

8. Strassmayr C, Matanov A, Priebe $\mathrm{S}$, Barros H, Canavan $\mathrm{R}$, et al. (2012) Mental health care for irregular migrants in Europe: Barriers and how they are overcome. BMC Public Health 12: 367.

9. Bayram N, Thorburn D, Demirhan H, Bilgel N (2007) Quality of life among Turkish immigrants in Sweden. Qual Life Res 16(8): 1319-1333.

10. Mui AC, Kang SY, Kang D, Domanski MD (2007) English language proficiency and health-related quality of life among Chinese and Korean immigrant elders. Health Soc Work 32(2): 119-127.

11. Koochek A, Montazeri A, Johansson SE, Sundquist J (2007) Health-related quality of life and migration: a cross-sectional study on elderly Iranians in Sweden. Health Qual Life Outcomes 5: 60.

12. Nesterko Y, Braehler E, Grande G, Glaesmer H (2012) Life satisfaction and health-related quality of life in immigrants and native-born Germans: the role of immigration-related factors. Quality of Life Research 22(5): 1005-1013.

13. Daher AM, Ibrahim HS, Daher TM, Anbori AK (2011) Health related quality of life among Iraqi immigrants settled in Malaysia. BMC Public Health 11: 407.

14. Poppe C, Crombez G, Hanoulle I, Vogelaers D, Petrovic M (2012) Mental quality of life in chronic fatigue is associated with an accommodative coping style and neuroticism: a path analysis. Quality of Life Research 21(8): 1337-1345.

15. Carver CS, Connor Smith J (2010) Personality and coping. Annual review of psychology 61: 679-704. 


\section{Nursing \& Healthcare International Journal}

16. Connor Smith JK, Flachsbart C (2007) Relations between personality and coping: a meta-analysis. J Pers Soc Psychol 93(6): 1080-1107.

17. Pantzer K, Rajmil L, Tebé C, Codina F, Serra-Sutton V, et al. (2006) Health related quality of life in immigrants and native school aged adolescents in Spain. J Epidemiol Community Health 60(8): 694-98.

18. Chou FH, Chen PC, Liu R, Ho CK, Tsai KY, et al. (2010a) A comparison of quality of life and depression between female married immigrants and native married women in Taiwan. Social psychiatry and psychiatric epidemiology 45(9): 921-930.

19. Wong WK, Chou KL, Chow NW (2012) Correlates of Quality of Life in New Migrants to Hong Kong from Mainland China. Social indicators research 107(2): 373-391.

20. Khan MA, Richardson J (2009) Report on Health Related Quality of Life and Lifestyle of Bangladeshi Migrants in Melbourne: Use of Multi Attribute Instruments. Melbourne: Centre for Health Economics, Monash University.

21. Mier N, Ory MG, Zhan D, Conkling M, Sharkey JR, et al. (2008) Health-related quality of life among Mexican Americans living in colonias at the Texas-Mexico border. Soc Sci Med 66(8): 1760-1771.

22. Maryam T, Arab M, Montazeri A (2008) Determinants of health-related quality of life in elderly in Tehran, Iran. BMC Public Health 8(1): 323.

23. Lasheras C, Patterson AM, Casado C, Fernandez S (2001) Effects of education on the quality of life, diet, and cardiovascular risk factors in an elderly Spanish community population. Exp Aging Res 27(3): 257 270 .

24. Wahl AK, Rustoen T, Hanestad BR, Lerdal A, Moum T (2004) Quality of life in the general Norwegian population, measured by the Quality of Life Scale (QOLS-N) Qual Life Res 13(5): 1001-1009.

25. UNDP (2011) Human Development Report 2011. Washington United Nations, United Nations Development Programme.

26. Australian Bureau of Statistics (2011) Community Information Summary, Canberra Australian Bureau of Statistics.
27. Alonso J, Ferrer M, Gandek B, Ware JE Jr, Aaronson NK (2004) Health-related quality of life associated with chronic conditions in eight countries: results from the International Quality of Life Assessment (IQOLA) Project. Qual Life Res 13(2): 283-298.

28. Langeland E, Wahl AK, Kristoffersen K, Nortvedt MW, Hanestad BR (2007) Quality of Life Among Norwegians with Chronic Mental Health Problems Living in the Community versus the General Population. Community Ment Health J 43(4): 321-339.

29. Baruth M, Wilcox S, Laken M, Bopp M, Saunders R (2008) Implementation of a faith-based physical activity intervention: Insights from church health directors. J Community Health 33(5): 304-312.

30. Campbell MK, Hudson MA, Resnicow K, Blakeney N, Paxton A, et al. (2007) Church-based health promotion interventions: evidence and lessons learned. Annu Rev Public Health 28: 213-234.

31. Coleman Brueckheimer K, Spitzer J, Koffman J (2009) Involvement of Rabbinic and communal authorities in decision-making by haredi Jews in the UK with breast cancer: An interpretative phenomenological analysis. Soc Sci Med 68(2): 323-333.

32. Peterson J, Atwood JR, Yates B (2002) Key Elements for Church-Based Health Promotion Programs: Outcome-Based Literature Review. Public Health Nursing 19(6): 401-411.

33. Burckhardt CS, Anderson KL (2003) The Quality of Life Scale (QOLS): reliability, validity, and utilization. Health Qual Life Outcomes 1(1): 60.

34. Liedberg GM, Burckhardt CS, Henriksson CM (2005) Validity and reliability testing of the Quality of Life Scale, Swedish version in women with fibromyalgiastatistical analyses. Scand J Caring Sci 19(1): 64-70.

35. Andrews FM, Crandall R (1976) The validity of measures of self-reported well-being. Social indicators research 3(1): 1-19.

36. Guillemin F, Bombardier C, Beaton D (1993) Crosscultural adaptation of health-related quality of life measures: literature review and proposed guidelines. J Clin Epidemiol 46(12): 1417-1432.

37. Fernandez RS, Salamonson Y, Griffiths R, Juergens C, Davidson P (2008) Sociodemographic predictors and 


\section{Nursing \& Healthcare International Journal}

reasons for participation in an outpatient cardiac rehabilitation programme following percutaneous coronary intervention. Int J Nurs Pract 14(3): $237-$ 242.

38. Can G, Durna Z, Aydiner A (2010) The validity and reliability of the Turkish version of the Quality of Life Index [QLI] (Cancer version) Eur J Oncol Nurs 14(4): 316-321.

39. Minas H, Klimidis S, Kokanovic R (2007) Depression in multicultural Australia: Policies, research and services. Australia and New Zealand Health Policy $4(1): 16$.

40. Voss U, Tuin I (2008) Integration of immigrants into a new culture is related to poor sleep quality. Health Qual Life Outcomes 6(1): 61.

41. Maryam R (2013) From Expression to Collaboration: The role of art in fostering participation and social inclusion among CALD communities in Australian Mosaic. Perth: Federation of Ethnic Communities' Councils of Australia (FECCA).

42. Leckey J (2011) The therapeutic effectiveness of creative activities on mental well-being: a systematic review of the literature. J Psychiatr Ment Health Nurs 18(6): 501-509.

43. Chou YC, Pu CY, Kröger T, Fu LY (2010b) Caring, employment, and quality of life: Comparison of employed and nonemployed mothers of adults with intellectual disability. Am J Intellect Dev Disabil 115(5): 406-420.

44. Phaswana-Mafuya N, Peltzer K, Chirinda W, Kose Z, Hoosain E (2013) Self-rated health and associated factors among older South Africans: evidence from the study on global ageing and adult health. Global Health Action 6(1): 19880.

45. Low G, Molzahn AE (2007) Predictors of quality of life in old age: A cross-validation study. Res Nurs Health $30(2): 141-150$.

46. Nesterko Y, Braehler E, Grande G, Glaesmer H (2013) Life satisfaction and health-related quality of life in immigrants and native-born Germans: the role of immigration-related factors. Qual Life Res 22(5): 1005-1013.

47. Knabe A, Rätzel S, Schöb R, Weimann J (2010) Dissatisfied with Life but Having a Good Day: Time-use and Well-being of the Unemployed. The Economic Journal 120(547): 867-889.

48. Pamela G (2010) Social desirability bias. Wiley International Encyclopedia of Marketing.

49. Kopel E, Keinan Boker L, Enav T, Dichtiar R, Shohat T (2013) Cigarette Smoking and Correlates Among Ultra-Orthodox Jewish Males. Nicotine Tob Res 15(2): 562-566. 